\title{
Stability, Adaptability Analysis of Wheat Genotypes by AMMI with BLUP for Irrigated Late Sown Trials in North Western Plains Zone
}

\author{
Ajay Verma* and G. P. Singh \\ ICAR-Indian Institute of Wheat \& Barley Research, Karnal, Haryana, India \\ *Corresponding author
}

\section{Keywords \\ AMMI model, MASV, WAASB, \\ SI, Biplotgraphical analysis}

\section{Article Info}

Accepted:

10 January 2021

Available Online:

1 February 2021

\section{A B S T R A C T}

Effects of the environment, GxE interaction, and genotypes were highly significant by AMMI analysis for wheat genotypes evaluated under irrigated late sown multi-location trials in the North-Western Plains zone of the country during 2018-19 and 2019-20. The preference order of genotypes had been altered with the utilization of more number of IPCA's in AMMI and WAASB based measures. Genotypes WH1124, PBW752, PBW771 had marked by adaptability and superiority indexes. Two significant PC has explained $83.6 \%$ of the total variation in the Biplot analysis and adaptability measures placed in a separate quadrant. A separate cluster of Superiority Indexes had also observed while considering various averages. This group maintained the right angles with a group of MASV \& MASV1 measures. The second year of study had reflected that HD3059, UP3033, HD3334, and PBW771wheat genotypes were selected by adaptability and superiority indexes of genotypes. About $74 \%$ of the total variation accounted for by PC1 \& PC2 in Biplot analysis. Adaptability measures expressed strong bondage and were placed in a different quadrant. The cluster of Superiority indexes of wheat genotypes seen in a different quadrant.

\section{Introduction}

Prime objective under multi location crop breeding trials is to understand the GxE interaction efficiently and ways to exploit it for selection of highly productive genotypes (Agahi et al., 2020). Good number of statistical analytic tools has been developed to estimate GxE interactions (Ajay et al., 2020). Mostly AMMI analysis is used as it considers fixed-effect of genotypes under statistical model (Bocianowski et al., 2019). Recently it may be reasonable to consider genotypes, environments, or both to be random effects in mixed model approach (Piepho et al., 2008). Currently, the BLUP mixed model is the most adequate procedure for genetic evaluation in breeding, and it has become necessary for understanding the $\mathrm{G} \times \mathrm{E}$ interaction (Ashwini et al., 2021). Methods convert measures of adaptability and stability along with yield into a single measure, such as of Annicchiarico (1992) and Lin and Binns (1988), were preferred in practical use as allowed a simpler interpretation, especially when number of genotypes were elevated at number of 
locations (Kang 1993; Rao and Prabhakaran 2005; Farshadfar, 2008; Farshadfar et al., 2011).Taking into account the benefits of AMMI and BLUP, a measure Superiority Index had been put forward for stability and adaptability of genotypes (Olivoto et al., 2019).The present study dealt with the merits of the analysis of GxE interaction and yield stability through AMMI with BLUP techniques for wheat genotypes evaluated under multi locations trials in the North Western Plains zone of India.

\section{Materials and Methods}

The states of Punjab, Haryana, Delhi, Rajasthan (except Kota and Udaipur divisions), Western Uttar Pradesh (except Jhansi division and hilly areas), parts of Jammu and Kashmir (Jammu and Kathua districts) and parts of Himachal Pradesh (Paonta Valley and Una districts) categorised as the North Western Plains Zone of India. During cropping seasons of 2018-19 and 2019-20 ten promising wheat genotypes in advanced trials evaluated at fourteen major locations of the zone and nine genotypes at thirteen locations were evaluated respectively. Field trials were conducted at research centers in randomized complete block designs with three replications. Recommended agronomic practices were followed to harvest good yield. Details of locations and genotype parentage were reflected in tables $1 \& 2$ for ready reference.

Stability measure as Weighted Average of Absolute Scores calculated as

$\mathrm{WAASB}=\sum_{k=1}^{p}\left|I P C A_{i k} \times E P_{k}\right| / \sum_{k=1}^{p} E P_{k}$; WAASB $_{i}$ was the weighted average of absolute scores of the $i$ th genotype (or environment); $\mathrm{IPCA}_{i k}$ the score of the $i$ th genotype (or environment) in the $k$ th IPCA, and $\mathrm{EP}_{k}$ was the amount of the variance explained by the $k$ th IPCA. Superiority index allowed variable weights to yield and stability measure (WAASB) to select genotypes that combine high performance and stability as SI $\underline{\left(r G_{i} \times \theta_{Y}\right)+\left(r W W_{i} \times \theta_{S}\right)}$

$=\quad\left(\theta_{Y}+\theta_{S}\right) \quad$; where $r G_{i}$ and $r W_{i}$ were the rescaled values for yield and WAASB, respectively, for the $i$ th genotype; $G_{i}$ and $W_{i}$ were the yield and the WAASB values for $i$ th genotype. SI superiority index for the $i$ th genotype that weighted between yield and stability, and $\theta Y$ and $\theta S$ were the weights for yield and stability assumed to be of order 65 and 35 respectively in this study,

\begin{tabular}{|c|c|c|}
\hline $\begin{array}{l}\text { Mohamadi and } \\
\text { Amri, } 2008\end{array}$ & $\begin{array}{c}\text { Geometric } \\
\text { Adaptability Index }\end{array}$ & $\mathbf{G A I}=\sqrt[n]{\prod_{\mathrm{k}=1}^{\mathrm{n}} \overline{\mathrm{X}}_{\mathrm{k}}}$ \\
\hline Zali et al., 2012 & $\begin{array}{l}\text { Modified AMMI } \\
\text { stability Value }\end{array}$ & MAASV $=\sqrt{\sum_{n=1}^{x-1} \frac{S S I P C_{n}}{S S I P C_{n+1}}\left(P C_{n}\right)^{2}+\left(P C_{n+1}\right)^{2}}$ \\
\hline Ajay et al., 2019 & MASV1 & MASV1 $=\sqrt{\left.\sum_{n=1}^{N-1} \frac{S S I P C_{n}}{S S I P C_{n+1}} P C_{n}\right)^{2}+\left(P C_{n+1}\right)^{2}}$ \\
\hline $\begin{array}{l}\text { Resende and } \\
\text { Durate, 2007 }\end{array}$ & $\begin{array}{l}\text { Relative performance of } \\
\text { genotypic values across } \\
\text { environments }\end{array}$ & $P R V G_{i j}=V G_{i j} / V_{i}$ \\
\hline $\begin{array}{l}\text { Resende and } \\
\text { Durate, 2007 }\end{array}$ & $\begin{array}{l}\text { Harmonic mean of } \\
\text { Relative performance of } \\
\text { genotypic values }\end{array}$ & MHPRVG $_{i .}=\underset{\sum_{j=1}^{k} \frac{1}{p R V G_{i j}}}{\text { Number of environments / }}$ \\
\hline $\begin{array}{l}\text { Oliveto et al., } \\
\quad 2019\end{array}$ & Superiority Index & 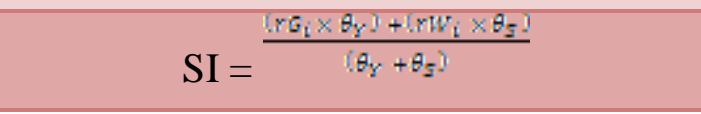 \\
\hline
\end{tabular}


AMMISOFT version 1.0, available at https://scs.cals.cornell.edu/people/ hughgauch/ utilized for AMMI analysis of wheat genotypes evaluated under multi-location trials in the Peninsular Zone and further analysis carried out by SAS software version 9.3. Stability measures had been compared with recent analytic measures of adaptability calculated as the relative performance of genetic values (PRVG) and harmonic mean based measure of the relative performance of the genotypic values (MHPRVG) for the simultaneous analysis of stability, adaptability, and yield (Mendes et al., 2012).

\section{Results and Discussion}

\section{First-year of study (2018-19)}

AMMI based stability measures permit to evaluate of the stability of genotypes after the reduction of the noise from the $\mathrm{GxE}$ interaction effects. Highly significant effects of the environment (E), GxE interaction, and genotypes (G) were observed (Table 3). Analysis observed the greater contribution of environments, GxE interactions, and genotypes to the total sum of squares (SS) as compared to the residual effects. Further SS attributable to $\mathrm{GxE}$ interactions was partitioned as attributed to GxE interactions Signal and GxE interactions Noise. AMMI analysis is appropriate for data sets where-in SS due to were of magnitude at least of due to additive genotype main effects (Gauch 2013). The SS for GxE interactions Signal was higher compared to genotype main effects, indicated appropriateness of AMMI analysis. Environment explained about $42.8 \%$ of the total sum of squares due to treatments. Moreover up to $80 \%$ contribution of environmental effects reported in various crops (Oyekunle et al., 2017). Genotypes explained only $15.6 \%$ of the total sum of squares, whereas GxE interaction accounted for $24.6 \%$ of treatment variations in yield. Further interaction was divided into four significant multiplicative terms explained 97 $\%$ and the remaining $2.8 \%$ was discarded residual.

\section{Ranking of genotypes vis-à-vis number of IPCA's}

The IPCA scores of genotypes in the AMMI analysis are an indication of stability or adaptability over environments. The greater the IPCA scores, the more specific adapted is a genotype to certain locations. The more the IPCA scores approximate to zero, the more stable or adapted the genotypes is overall the locations. The ranking of genotype as per absolute IPCA-1 scores wereWH1124, PBW752 (Table 4). While for IPCA-2, genotypes DBW173, WH1021 would be of choice. Values of IPCA-3 favoured DBW173, WH1021 wheat genotypes. As per IPCA-4, HD3059, PBW752 genotypes would be of stable performance. Analytic measures of adaptability MASV and MASV1 consider all significant IPCAs of the analysis. Values of MASV1 identified genotypes WH1124, PBW771 would express stable yield whereas genotypes PBW771, WH1124 be of stable performance by MASV measure respectively (Ajay et al., 2019).

To study how the ranks of genotype are altered with different numbers of IPCA used in the WAASB estimation, the genotype's ranks were obtained considering the WAASB estimated with $1,2, \ldots, 4$ IPCA. When using only one IPCA, WAASB = |IPCA1|. The ranking was increasing; so, the genotype with the smallest WAASB value had the first-order rank. Preferences of genotypes had varied with increasing use of many IPCA as WH1124, PBW752 based on W1 whereas WH1124, HD3059 as per W2 values while WH1124, HD3059 by values of W3 (Table 
5). Stability measure WAASB based on all significant IPCA's settled for WH1124, HD3059 genotypes for considered locations of the zone for stable high yield. The genotype ranking was altered by the extent to which IPCAs were included in the WAASB estimation. This reinforced the benefits of using the WAASB index since it captured the variations of all IPCAs to compute the stability (Olivoto et al., 2019).

\section{Stable productive genotypes by AMMI \& BLUP tools}

An average yield selected PBW771, PBW752, wheat genotypes (Table 6). This method is simple, but not fully exploiting all information contained in the dataset. A geometric mean is used to evaluate the adaptability of genotypes and genotypes with high GM will be desirable. Geometric mean observed PBW771, PBW752 were top-ranked genotypes. As proposed by Resende (2007), a method to rank genotypes considering the yield and stability simultaneously is the harmonic mean of genetic values (HMGV). In the context of mixed models, the Harmonic Mean of Genotypic Values were calculated as genotypes with greater values would be recommended. Harmonic Mean of yield expressed higher values for PBW771, PBW752, genotypes. For stability analysis of the Harmonic Mean of Relative Performance of Genotypic Values (HMRPGV) method, the genotypes can be simultaneously sorted by genotypic values (yield) and stability using the harmonic means of the yield so that the smaller the standard deviation of genotypic performance among the locations. Values of HMRPGV ranked PBW771, PBW752 the genotypes' performance among the locations. When considering the yield and adaptability simultaneously, the recommended approach is the relative performance of genetic values (RPGV) over crop years. Relative Performance of Genotypic Values had settled for PBW771, PBW752 wheat genotypes.

Table.1 Details of locations and parentage of evaluated wheat genotypes (2018-19)

\begin{tabular}{|l|l|l|c|c|c|c|}
\hline Code & Genotype & Parentage & Location & Latitude & Longitude & $\begin{array}{c}\text { Mean } \\
\text { sea } \\
\text { level }\end{array}$ \\
\hline G 1 & PBW 752 & (PBW621/4/PBW343//YR10/6*AVOCET/3/3*PBW343/5/PBW621) & Delhi & $28^{\circ} 4^{\prime} \mathrm{N}$ & $77^{\circ} 13^{\prime} \mathrm{E}$ & 228 \\
\hline G 2 & DBW 173 & (KAUZ/AA//KAUZ//PBW62) & Bawal & $28^{\circ} 10^{\prime} \mathrm{N}$ & $76^{\circ} 50^{\prime} \mathrm{E}$ & 266 \\
\hline G 3 & WH 1021 & (NYOT95/SONAK) & Hisar & $29^{\circ} 10^{\prime} \mathrm{N}$ & $75^{\circ} 46^{\prime} \mathrm{E}$ & 229 \\
\hline G 4 & HD 3059 & (KAUZ//ALTAR84/AOS/3/MILAN/KAUZ/4/HUITES) & Karnal & $29^{\circ} 43^{\prime} \mathrm{N}$ & $70^{\circ} 58^{\prime} \mathrm{E}$ & 245 \\
\hline G 5 & WH 1124 & (MUNIA/CHTO//AMSEL) & Rohtak & $28^{\circ} 53^{\prime} \mathrm{N}$ & $76^{\circ} 35^{\prime} \mathrm{E}$ & 222.5 \\
\hline G 6 & PBW 771 & (PBW550//YR15/6*AVOCET/3/2*PBW550) & Shikohpur & $28^{\circ} 36^{\prime} \mathrm{N}$ & $76^{\circ} 98^{\prime} \mathrm{E}$ & 259 \\
\hline & & & Jammu & $32^{\circ} 40^{\prime} \mathrm{N}$ & $74^{\circ} 54^{\prime} \mathrm{E}$ & 356 \\
\hline & & & Ludhiana & $30^{\circ} 54^{\prime} \mathrm{N}$ & $75^{\circ} 48^{\prime} \mathrm{E}$ & 247 \\
\hline & & & Kapurthala & $31^{\circ} 22^{\prime} \mathrm{N}$ & $75^{\circ} 22^{\prime} \mathrm{E}$ & 229 \\
\hline & & & Gurdaspur & $30^{\circ} 02^{\prime} \mathrm{N}$ & $75^{\circ} 24^{\prime} \mathrm{E}$ & 265 \\
\hline & & & Bathinda & $30^{\circ} 09^{\prime} \mathrm{N}$ & $74^{\circ} 55^{\prime} \mathrm{E}$ & 211 \\
\hline & & & Faridkot & $30^{\circ} 40^{\prime} \mathrm{N}$ & $74^{\circ} 04{ }^{\prime} \mathrm{E}$ & 200 \\
\hline & & & Durgapura & $26^{\circ} 51^{\prime} \mathrm{N}$ & $75^{\circ} 47^{\prime} \mathrm{E}$ & 390 \\
\hline & & & Tabiji & $26^{\circ} 35^{\prime} \mathrm{N}$ & $74^{\circ} 61^{\prime} \mathrm{E}$ & 508 \\
\hline & & & Sriganganagar & $29^{\circ} 66^{\prime} \mathrm{N}$ & $75^{\circ} 53^{\prime} \mathrm{E}$ & 175.6 \\
\hline & & & Alwar & $27^{\circ} 7^{\prime} \mathrm{N}$ & $76^{\circ} 11^{\prime} \mathrm{E}$ & 271 \\
\hline & & & Nagina & $29^{\circ} 28^{\prime} \mathrm{N}$ & $78^{\circ} 32^{\prime} \mathrm{E}$ & 245 \\
\hline & & & Bulandshahr & $28^{\circ} 40^{\prime} \mathrm{N}$ & $77^{\circ} 84^{\prime} \mathrm{E}$ & 195 \\
\hline & & & Ujhani & $28^{\circ} 06^{\prime} \mathrm{N}$ & $79^{\circ} 34^{\prime} \mathrm{E}$ & \\
\hline & & & Pantnagar & $29^{\circ} 02^{\prime} \mathrm{N}$ & $79^{\circ} 48^{\prime} \mathrm{E}$ & 243.8 \\
\hline & & & Kashipur & $29^{\circ} 21^{\prime} \mathrm{N}$ & $78^{\circ} 96^{\prime} \mathrm{E}$ & 218 \\
\hline
\end{tabular}


Table.2 Details of locations and parentage of evaluated wheat genotypes (2019-20)

\begin{tabular}{|c|c|c|c|c|c|c|}
\hline Code & Genotype & Parentage & Location & Latitude & Longitude & $\begin{array}{l}\text { Mean } \\
\text { sea } \\
\text { level }\end{array}$ \\
\hline G 1 & HD3334 & (DBW 50/WR2502) & Delhi & $28^{\circ} 4^{\prime} \mathrm{N}$ & $77^{\circ} 13^{\prime} \mathrm{E}$ & 228 \\
\hline G 2 & HD3059 & (KAUZ//ALTAR84/AOS/3/MILAN/KAUZ/4/HUITES) & Hisar & $29^{\circ} 10^{\prime} \mathrm{N}$ & $75^{\circ} 46^{\prime} \mathrm{E}$ & 229 \\
\hline G 3 & HD3332 & (PFAU/SERI.1B) & Karnal & $29^{\circ} 43^{\prime} \mathrm{N}$ & $70^{\circ} 58^{\prime} \mathrm{E}$ & 245 \\
\hline G 4 & DBW173 & (KAUZ/AA//KAUZ//PBW602) & Rohtak & $28^{\circ} 53^{\prime} \mathrm{N}$ & $76^{\circ} 35^{\prime} \mathrm{E}$ & 222.5 \\
\hline G 5 & WH1021 & (NYOT95/SONAK) & Shikohpur & $28^{\circ} 36^{\prime} \mathrm{N}$ & $76^{\circ} 98^{\prime} \mathrm{E}$ & 259 \\
\hline G 6 & PBW811 & (BECARD/2*FRNCLN) & Ludhiana & $30^{\circ} 54^{\prime} \mathrm{N}$ & $75^{\circ} 48^{\prime} \mathrm{E}$ & 247 \\
\hline G 7 & DBW291 & (KRL236/DBW16) & Kapurthala & $31^{\circ} 22^{\prime} \mathrm{N}$ & $75^{\circ} 22^{\prime} \mathrm{E}$ & 229 \\
\hline G 8 & WH1264 & (P12256/P12332//WH1142) & Gurdaspur & $30^{\circ} 02^{\prime} \mathrm{N}$ & $75^{\circ} 24^{\prime} \mathrm{E}$ & 265 \\
\hline G 9 & PBW812 & (BWL 0762/PBW621//HD 3086) & Bathinda & $30^{\circ} 09^{\prime} \mathrm{N}$ & $74^{\circ} 55^{\prime} \mathrm{E}$ & 211 \\
\hline G 10 & JKW261 & (ISENGRAIN/KBIRD//MUNAL\#1) & Faridkot & $30^{\circ} 400^{\prime} \mathrm{N}$ & $74^{\circ} 04^{\prime} \mathrm{E}$ & 200 \\
\hline G 11 & DBW290 & $\begin{array}{l}\text { (QUAIU\#1/5/KIRITATI/4/2*SERI.1B*2/3/KAUZ*2/BO } \\
\text { W//KAUZ/6/BECARD(35th ESWYT 140) }\end{array}$ & Durgapura & $26^{\circ} 51^{\prime} \mathrm{N}$ & $75^{\circ} 47^{\prime} \mathrm{E}$ & 390 \\
\hline G 12 & PBW771 & (PBW550//YR15/6*AVOCET/3/2*PBW550) & $\begin{array}{l}\text { Srigangana } \\
\text { gar }\end{array}$ & $29^{\circ} 66^{\prime} \mathrm{N}$ & $75^{\circ} 53^{\prime} \mathrm{E}$ & 175.6 \\
\hline G 13 & PBW813 & $\begin{array}{l}\text { (KRICHAUFF/4/2*BW9250*3//Yr10/6*Avocet/3/BW } \\
9250 * 3 / / Y r \text { 15/6*Avocet }\end{array}$ & Nagina & $29^{\circ} 28^{\prime} \mathrm{N}$ & $78^{\circ} 32^{\prime} \mathrm{E}$ & 245 \\
\hline G 14 & HD3331 & $\begin{array}{l}\text { (31stESWYT- } \\
\text { 135/3/HD2329/WR544//PBW343/NW3041) }\end{array}$ & $\begin{array}{l}\text { Bulandsha } \\
\mathrm{hr}\end{array}$ & $28^{\circ} 40 ' \mathrm{~N}$ & $77^{\circ} 84^{\prime} \mathrm{E}$ & 195 \\
\hline G 15 & HD3298 & (CL1449/PBW343//CL882/HD2009) & Ujhani & $28^{\circ} 06^{\prime} \mathrm{N}$ & $79^{\circ} 34^{\prime} \mathrm{E}$ & \\
\hline G 16 & WH1124 & (MUNIA/CHTO//AMSEL) & $\begin{array}{l}\text { Modipura } \\
\mathrm{m}\end{array}$ & $29^{\circ} 05^{\prime} \mathrm{N}$ & $77^{\circ} 70^{\prime} \mathrm{E}$ & 226 \\
\hline \multirow[t]{2}{*}{ G 17} & UP3033 & (NIAW 1544/UP2763) & Pantnagar & $29^{\circ} 02 \mathrm{~N}$ & $79^{\circ} 48^{\prime} \mathrm{E}$ & 243.8 \\
\hline & & & Kashipur & $29^{\circ} 21^{\prime} \mathrm{N}$ & $78^{\circ} 96^{\prime} \mathrm{E}$ & 218 \\
\hline
\end{tabular}

Table.3 AMMI analysis of wheat genotypes for irrigated late sown multi location trials during 2018-19\& 2019-20

\begin{tabular}{|l|l|l|l|l|l|l|}
\hline Source & $\begin{array}{l}\text { Degree of } \\
\text { freedom } \\
\mathbf{1 8 - 1 9}\end{array}$ & $\begin{array}{l}\text { Degree of } \\
\text { freedom } \\
\mathbf{1 9 - 2 0}\end{array}$ & $\begin{array}{l}\text { Mean Sum } \\
\text { of Squares } \\
\mathbf{1 8 - 1 9}\end{array}$ & $\begin{array}{l}\text { Mean Sum } \\
\text { of Squares } \\
\mathbf{1 9 - 2 0}\end{array}$ & $\begin{array}{l}\text { Level of } \\
\text { significance } \\
\mathbf{1 8 - 1 9}\end{array}$ & $\begin{array}{l}\text { Level of significance } \\
\mathbf{1 9 - 2 0}\end{array}$ \\
\hline Treatments & 125 & 305 & 250.5698 & 214.92 & $.0000000 * * *$ & $.0000000 * * *$ \\
\hline Genotypes (G) & 5 & 16 & 1178.312 & 556.53 & $.0000000 * * *$ & $.0000000 * * *$ \\
\hline Environments (E) & 20 & 17 & 808.3152 & 1744.59 & $.0000000 * * *$ & $.0000000 * * *$ \\
\hline Interactions GxE & 100 & 272 & 92.63365 & 99.22 & $.0000000 * * *$ & $.0000000 * * *$ \\
\hline IPC1 & 24 & 32 & 206.8943 & 269.40 & $.0000000 * * *$ & $.0000000 * * *$ \\
\hline IPC2 & 22 & 30 & 86.01315 & 158.63 & $.0000000 * * *$ & $.0000000 * * *$ \\
\hline IPC3 & 20 & 28 & 63.69132 & 111.87 & $.0000001 * * *$ & $.0000000 * * *$ \\
\hline IPC4 & 18 & 26 & 47.51667 & 99.02 & $.0014130 * *$ & $.0000000 * * *$ \\
\hline IPC5 & & 24 & & 98.97 & & $.0000000 * * *$ \\
\hline IPC6 & & 22 & & 59.39 & & $.0000000 * * *$ \\
\hline IPC7 & & 20 & & 57.93 & & $.0000000 * * *$ \\
\hline Residual & 16 & 90 & 17.28031 & 34.01 & 0.4364813 & $.0000000 * * *$ \\
\hline Error & 378 & 918 & 16.97693 & 11.07 & & \\
\hline Total & 503 & 1223 & 75.02685 & 61.91 & & \\
\hline
\end{tabular}


Table.4 Modified AMMI stability values as per significant IPCA’s 2018-19

\begin{tabular}{|c|c|c|c|c|c|c|c|c|c|c|c|c|c|c|c|c|c|}
\hline Genotype & IPCA1 & IPCA2 & IPCA3 & IPCA4 & MASV1 & MASV & W1 & W2 & W3 & WAASB & $\mathbf{R}_{\text {IPCA1 }}$ & $\mathbf{R}_{\text {MASV1 }}$ & $\mathbf{R}_{\text {MASV }}$ & $\mathbf{R}_{\mathrm{W} 1}$ & $\mathbf{R}_{\mathrm{W} 2}$ & $\mathbf{R}_{\mathrm{W} 3}$ & $\mathbf{R}_{\text {WAASB }}$ \\
\hline PBW 752 & -0.344 & -3.991 & -0.673 & -0.210 & 7.307 & 6.408 & 0.344 & 1.415 & 1.282 & 1.156 & 2 & 4 & 5 & 2 & 4 & 3 & 3 \\
\hline DBW 173 & 2.945 & -0.239 & 0.030 & -0.842 & 7.786 & 4.859 & 2.945 & 2.150 & 1.772 & 1.662 & 5 & 5 & 3 & 5 & 5 & 5 & 5 \\
\hline WH 1021 & -4.868 & 0.570 & 0.208 & 0.374 & 12.826 & 7.953 & 4.868 & 3.606 & 2.999 & 2.691 & 6 & 6 & 6 & 6 & 6 & 6 & 6 \\
\hline HD 3059 & 0.724 & 0.630 & 3.513 & -0.203 & 6.682 & 5.756 & 0.724 & 0.696 & 1.200 & 1.082 & 3 & 3 & 4 & 3 & 2 & 2 & 2 \\
\hline WH 1124 & 0.055 & 1.992 & -1.903 & -2.138 & 5.383 & 4.843 & 0.055 & 0.624 & 0.852 & 1.003 & 1 & 1 & 2 & 1 & 1 & 1 & 1 \\
\hline PBW 771 & 1.488 & 1.038 & -1.175 & 3.019 & 5.680 & 4.587 & 1.488 & 1.356 & 1.324 & 1.523 & 4 & 2 & 1 & 4 & 3 & 4 & 4 \\
\hline
\end{tabular}

$\mathrm{R}_{\mathrm{W} 1}, \mathrm{R}_{\mathrm{W} 2}, \mathrm{R}_{\mathrm{W} 3}, \mathrm{R}_{\mathrm{W} 4}, \mathrm{R}_{\mathrm{W} 5}, \mathrm{R}_{\mathrm{W} 6}, \mathrm{R}_{\mathrm{WAASB}}=$ Rank of genotypes as per number of IPCA's in WAASB values

Table.5 Superiority index and analytic adaptability measures based on BLUP's of genotypes 2018-19

\begin{tabular}{|c|c|c|c|c|c|c|c|c|c|c|c|c|c|c|c|c|}
\hline Genotype & $\mathbf{A M u}$ & $\mathbf{R k}$ & SI au & $\mathbf{R k}$ & GMu & $\mathbf{R k}$ & SI gu & $\mathbf{R k}$ & HMu & $\mathbf{R k}$ & SI hu & $\mathbf{R k}$ & MHRPGVu & $\mathbf{R k}$ & RPGVu & Rk \\
\hline PBW 752 & 49.64 & 2 & 88.71 & 3 & 49.24 & 2 & 89.57 & 2 & 48.84 & 2 & 90.70 & 2 & 1.040 & 2 & 1.047 & 2 \\
\hline DBW 173 & 48.00 & 4 & 66.92 & 4 & 47.38 & 4 & 66.41 & 4 & 46.73 & 4 & 65.90 & 5 & 1.001 & 4 & 1.007 & 4 \\
\hline WH 1021 & 41.34 & 6 & 0.00 & 6 & 40.74 & 6 & 0.00 & 6 & 40.16 & 6 & 0.00 & 6 & 0.854 & 6 & 0.873 & 6 \\
\hline HD 3059 & 45.89 & 5 & 64.54 & 5 & 45.47 & 5 & 65.45 & 5 & 45.04 & 5 & 66.42 & 4 & 0.961 & 5 & 0.966 & 5 \\
\hline WH 1124 & 49.50 & 3 & 90.89 & 1 & 48.96 & 3 & 90.81 & 1 & 48.41 & 3 & 90.95 & 1 & 1.036 & 3 & 1.039 & 3 \\
\hline PBW 771 & 50.83 & 1 & 89.22 & 2 & 50.31 & 1 & 89.22 & 3 & 49.74 & 1 & 89.22 & 3 & 1.064 & 1 & 1.069 & 1 \\
\hline
\end{tabular}

$\mathrm{AMu}, \mathrm{GMu}, \mathrm{HMu}=$ Arithmetic, Geometric, Harmonic Mean for BLUP values; SI au, SI gu, SI hu = Superiority index as per Arithmetic, Geometric, Harmonic Mean; RPGVu, MHRPGVu = Relative performance and Harmonic mean of Relative Performance as per BLUP of genotypes; Rk = Rank of genotypes 
Table.6 Loadings of BLUP based stability measures as per two significant PC's (2018-19)

\begin{tabular}{|l|l|l|}
\hline Measure & PC1 & PC2 \\
\hline IPCA1 & 0.2156 & -0.0297 \\
\hline IPCA2 & -0.0247 & -0.1598 \\
\hline IPCA3 & -0.0852 & -0.5192 \\
\hline IPCA4 & -0.0112 & 0.4375 \\
\hline MASV1 & -0.2668 & 0.1137 \\
\hline MASV & -0.2320 & -0.0195 \\
\hline W1 & -0.2409 & 0.2502 \\
\hline W2 & -0.2390 & 0.3333 \\
\hline W3 & -0.2603 & 0.2209 \\
\hline WAASB & -0.2459 & 0.3098 \\
\hline AMu & 0.2647 & 0.1977 \\
\hline SI au & 0.2740 & 0.0190 \\
\hline GMu & 0.2655 & 0.1891 \\
\hline SI gu & 0.2738 & 0.0135 \\
\hline HMu & 0.2662 & 0.1797 \\
\hline SI hu & 0.2736 & 0.0075 \\
\hline RPGVu & 0.2640 & 0.2040 \\
\hline MHRPGVu & 0.2667 & 0.1775 \\
\hline 83.68 & 73.62 & 10.06 \\
\hline
\end{tabular}

Table.7 Modified AMMI stability values as per significant IPCA's 2019-20

\begin{tabular}{|l|l|l|l|l|l|l|l|l|l|l|l|l|}
\hline Genotype & IPCA1 & IPCA2 & IPCA3 & IPCA4 & IPCA5 & IPCA6 & IPCA7 & MASV1 & MASV & R $_{\text {IPCA1 }}$ & R $_{\text {MASV1 }}$ & R $_{\text {MASV }}$ \\
\hline HD3334 & -0.225 & -0.603 & -1.448 & 1.331 & -1.072 & 0.276 & 0.191 & 3.947 & 3.575 & 3 & 2 & 3 \\
\hline HD3059 & 0.292 & -1.083 & -0.068 & 0.671 & -2.036 & 0.040 & -2.093 & 5.233 & 4.486 & 4 & 9 & 9 \\
\hline HD3332 & 0.094 & -1.551 & 0.973 & 1.842 & 0.688 & -0.361 & -0.720 & 4.535 & 4.168 & 2 & 5 & 7 \\
\hline DBW173 & 2.254 & -0.578 & -3.305 & -0.292 & -0.336 & 0.875 & 1.146 & 6.971 & 6.140 & 14 & 14 & 14 \\
\hline WH1021 & -4.025 & -1.290 & -1.221 & -2.027 & -0.680 & -1.456 & 0.980 & 8.891 & 7.223 & 17 & 17 & 17 \\
\hline PBW811 & -1.682 & 0.314 & 0.169 & 1.532 & -0.139 & 1.985 & 0.199 & 4.885 & 4.337 & 13 & 7 & 8 \\
\hline DBW291 & 0.832 & 1.175 & 0.099 & -0.157 & -0.361 & 0.528 & -0.013 & 2.848 & 2.401 & 7 & 1 & 1 \\
\hline WH1264 & -0.306 & -1.627 & 0.044 & 1.406 & 0.079 & -1.113 & -0.283 & 4.035 & 3.700 & 5 & 4 & 4 \\
\hline PBW812 & 1.228 & -1.052 & 0.245 & -1.182 & 0.628 & -0.675 & -1.120 & 3.973 & 3.450 & 12 & 3 & 2 \\
\hline JKW261 & 3.056 & 1.616 & 0.763 & -1.295 & -1.347 & -1.565 & -0.272 & 7.604 & 6.219 & 16 & 16 & 16 \\
\hline DBW290 & 0.962 & -1.073 & 1.007 & -2.158 & 0.215 & 2.306 & -0.097 & 5.637 & 5.289 & 10 & 10 & 12 \\
\hline PBW771 & 0.958 & -0.523 & 1.886 & 0.128 & 1.230 & 0.032 & 1.636 & 4.687 & 4.149 & 9 & 6 & 5 \\
\hline PBW813 & 0.882 & -1.351 & 0.027 & 0.070 & 1.826 & -0.683 & 0.923 & 4.987 & 4.155 & 8 & 8 & 6 \\
\hline HD3331 & 0.031 & 2.671 & 0.488 & 1.167 & -1.559 & -0.431 & 1.482 & 6.342 & 5.547 & 1 & 13 & 13 \\
\hline HD3298 & -1.125 & 2.480 & -1.709 & -0.696 & 1.906 & 0.220 & -1.594 & 7.147 & 6.177 & 11 & 15 & 15 \\
\hline WH1124 & -2.464 & 0.492 & 2.027 & -1.053 & -0.979 & 0.556 & -0.085 & 6.178 & 5.138 & 15 & 12 & 11 \\
\hline UP3033 & -0.762 & 1.983 & 0.024 & 0.714 & 1.936 & -0.535 & -0.279 & 5.734 & 4.824 & 6 & 11 & 10 \\
\hline
\end{tabular}


Table.8 Weighted average of absolute scores and ranks of wheat genotypes 2019-20

\begin{tabular}{|l|l|l|l|l|l|l|l|l|l|l|l|l|l|l|}
\hline Genotype & $\mathbf{W 1}$ & $\mathbf{W} 2$ & $\mathbf{W 3}$ & $\mathbf{W 4}$ & $\mathbf{W 5}$ & $\mathbf{W 6}$ & $\mathbf{W A A S B}$ & $\mathbf{R}_{\mathbf{W 1}}$ & $\mathbf{R}_{\mathbf{W 2}}$ & $\mathbf{R}_{\mathbf{W 3}}$ & $\mathbf{R}_{\text {W4 }}$ & $\mathbf{R}_{\mathbf{W 5}}$ & $\mathbf{R}_{\mathbf{W 6}}$ & $\mathbf{R}_{\mathbf{W A A S B}}$ \\
\hline HD3334 & 0.225 & 0.365 & 0.589 & 0.704 & 0.754 & 0.718 & 0.682 & 3 & 1 & 2 & 3 & 4 & 4 & 3 \\
\hline HD3059 & 0.292 & 0.585 & 0.478 & 0.508 & 0.713 & 0.663 & 0.760 & 4 & 2 & 1 & 1 & 3 & 2 & 4 \\
\hline HD3332 & 0.094 & 0.634 & 0.704 & 0.881 & 0.855 & 0.818 & 0.811 & 2 & 3 & 4 & 6 & 5 & 5 & 5 \\
\hline DBW173 & 2.254 & 1.633 & 1.979 & 1.718 & 1.532 & 1.484 & 1.461 & 14 & 14 & 15 & 15 & 13 & 14 & 14 \\
\hline WH1021 & 4.025 & 3.011 & 2.640 & 2.545 & 2.295 & 2.232 & 2.148 & 17 & 17 & 17 & 17 & 17 & 17 & 17 \\
\hline PBW811 & 1.682 & 1.175 & 0.967 & 1.054 & 0.931 & 1.010 & 0.955 & 13 & 11 & 8 & 11 & 8 & 10 & 9 \\
\hline DBW291 & 0.832 & 0.959 & 0.781 & 0.684 & 0.641 & 0.632 & 0.591 & 7 & 6 & 5 & 2 & 1 & 1 & 1 \\
\hline WH1264 & 0.306 & 0.796 & 0.640 & 0.759 & 0.668 & 0.701 & 0.672 & 5 & 4 & 3 & 5 & 2 & 3 & 2 \\
\hline PBW812 & 1.228 & 1.163 & 0.973 & 1.005 & 0.955 & 0.934 & 0.946 & 12 & 10 & 10 & 10 & 9 & 8 & 8 \\
\hline JKW261 & 3.056 & 2.522 & 2.157 & 2.024 & 1.933 & 1.906 & 1.795 & 16 & 16 & 16 & 16 & 16 & 16 & 16 \\
\hline DBW290 & 0.962 & 1.003 & 1.004 & 1.183 & 1.053 & 1.146 & 1.075 & 10 & 7 & 11 & 12 & 11 & 12 & 12 \\
\hline PBW771 & 0.958 & 0.797 & 1.023 & 0.884 & 0.931 & 0.864 & 0.916 & 9 & 5 & 12 & 7 & 7 & 7 & 7 \\
\hline PBW813 & 0.882 & 1.056 & 0.843 & 0.723 & 0.871 & 0.857 & 0.861 & 8 & 9 & 6 & 4 & 6 & 6 & 6 \\
\hline HD3331 & 0.031 & 1.009 & 0.901 & 0.942 & 1.025 & 0.981 & 1.015 & 1 & 8 & 7 & 9 & 10 & 9 & 11 \\
\hline HD3298 & 1.125 & 1.627 & 1.644 & 1.497 & 1.552 & 1.453 & 1.462 & 11 & 13 & 13 & 13 & 14 & 13 & 15 \\
\hline WH1124 & 2.464 & 1.733 & 1.794 & 1.679 & 1.586 & 1.509 & 1.412 & 15 & 15 & 14 & 14 & 15 & 15 & 13 \\
\hline UP3033 & 0.762 & 1.214 & 0.968 & 0.928 & 1.063 & 1.024 & 0.974 & 6 & 12 & 9 & 8 & 12 & 11 & 10 \\
\hline
\end{tabular}

Table.9 Superiority index and analytic adaptability measures based on BLUP's of genotypes 2019-20

\begin{tabular}{|c|c|c|c|c|c|c|c|c|c|c|c|c|c|c|c|c|}
\hline Genotype & AMu & Rk & SI au & $\mathbf{R k}$ & GMu & Rk & SI gu & Rk & HMu & Rk & SI hu & $\mathbf{R k}$ & MHRPGVu & $\mathbf{R k}$ & RPGVu & Rk \\
\hline HD3334 & 48.27 & 9 & 86.52 & 3 & 47.84 & 9 & 86.76 & 3 & 47.41 & 9 & 86.97 & 3 & 1.011 & 9 & 1.016 & 9 \\
\hline HD3059 & 49.68 & 2 & 93.68 & 1 & 49.19 & 2 & 92.91 & 1 & 48.72 & 3 & 92.34 & 1 & 1.034 & 3 & 1.049 & 2 \\
\hline HD3332 & 48.55 & 8 & 85.39 & 5 & 48.16 & 8 & 85.71 & 5 & 47.77 & 8 & 86.01 & 5 & 1.016 & 8 & 1.024 & 8 \\
\hline DBW173 & 49.43 & 3 & 76.37 & 9 & 49.15 & 3 & 76.88 & 9 & 48.84 & 2 & 77.24 & 9 & 1.037 & 2 & 1.044 & 3 \\
\hline WH1021 & 49.13 & 4 & 58.97 & 14 & 48.75 & 4 & 59.11 & 14 & 48.37 & 4 & 59.24 & 14 & 1.029 & 4 & 1.036 & 5 \\
\hline PBW811 & 47.75 & 10 & 77.09 & 8 & 47.34 & 10 & 77.69 & 8 & 46.92 & 11 & 78.20 & 8 & 0.998 & 11 & 1.007 & 10 \\
\hline DBW291 & 47.63 & 12 & 84.51 & 6 & 47.24 & 12 & 85.28 & 6 & 46.84 & 12 & 85.93 & 6 & 0.995 & 12 & 1.006 & 11 \\
\hline WH1264 & 45.13 & 15 & 66.87 & 12 & 44.56 & 15 & 67.83 & 12 & 44.00 & 15 & 68.69 & 12 & 0.938 & 15 & 0.950 & 15 \\
\hline PBW812 & 46.30 & 13 & 68.13 & 11 & 45.92 & 13 & 69.59 & 11 & 45.53 & 13 & 70.83 & 11 & 0.969 & 13 & 0.976 & 13 \\
\hline JKW261 & 46.25 & 14 & 48.73 & 16 & 45.70 & 14 & 49.23 & 16 & 45.17 & 14 & 49.81 & 16 & 0.964 & 14 & 0.971 & 14 \\
\hline DBW290 & 44.65 & 16 & 54.76 & 15 & 44.06 & 16 & 55.86 & 15 & 43.49 & 16 & 56.88 & 15 & 0.930 & 16 & 0.936 & 16 \\
\hline PBW771 & 49.07 & 5 & 86.34 & 4 & 48.68 & 5 & 86.38 & 4 & 48.27 & 5 & 86.40 & 4 & 1.024 & 6 & 1.038 & 4 \\
\hline PBW813 & 39.80 & 17 & 28.92 & 17 & 38.63 & 17 & 28.92 & 17 & 37.44 & 17 & 28.92 & 17 & 0.801 & 17 & 0.834 & 17 \\
\hline HD3331 & 48.70 & 7 & 81.76 & 7 & 48.28 & 7 & 81.83 & 7 & 47.84 & 7 & 81.81 & 7 & 1.019 & 7 & 1.026 & 7 \\
\hline HD3298 & 47.68 & 11 & 65.25 & 13 & 47.30 & 11 & 66.07 & 13 & 46.93 & 10 & 66.81 & 13 & 0.999 & 10 & 1.004 & 12 \\
\hline WH1124 & 48.97 & 6 & 74.50 & 10 & 48.48 & 6 & 74.09 & 10 & 47.99 & 6 & 73.68 & 10 & 1.024 & 5 & 1.029 & 6 \\
\hline UP3033 & 50.08 & 1 & 91.39 & 2 & 49.76 & 1 & 91.39 & 2 & 49.43 & 1 & 91.39 & 2 & 1.051 & 1 & 1.056 & 1 \\
\hline
\end{tabular}


Table.10 Loadings of BLUP based stability measures as per two significant PC's (2018-19)

\begin{tabular}{|l|l|l|}
\hline Measure & PC1 & PC2 \\
\hline IPCA1 & -0.0750 & 0.1120 \\
\hline IPCA2 & 0.0477 & -0.1184 \\
\hline IPCA3 & -0.0744 & 0.0515 \\
\hline IPCA4 & -0.2293 & -0.0677 \\
\hline IPCA5 & -0.0314 & 0.1262 \\
\hline IPCA6 & -0.0849 & -0.0325 \\
\hline IPCA7 & 0.0658 & 0.0260 \\
\hline MASV1 & 0.2898 & -0.0689 \\
\hline MASV & 0.2774 & -0.0710 \\
\hline W1 & 0.2906 & -0.0285 \\
\hline W2 & 0.3097 & -0.0227 \\
\hline W3 & 0.3102 & -0.0574 \\
\hline W4 & 0.3108 & -0.0588 \\
\hline W5 & 0.3140 & -0.0622 \\
\hline W6 & 0.3163 & -0.0475 \\
\hline WAASB & 0.3157 & -0.0543 \\
\hline AMu & 0.0068 & -0.3596 \\
\hline SI au & -0.1706 & -0.3044 \\
\hline GMu & 0.0068 & -0.3596 \\
\hline SI gu & -0.1721 & -0.3033 \\
\hline HMu & 0.0071 & -0.3592 \\
\hline SI hu & -0.1733 & -0.3021 \\
\hline RPGVu & 0.0024 & -0.3592 \\
\hline MHRPGVu & 0.0100 & -0.3593 \\
\hline 72.60 & 46.56 & 32.04 \\
\hline
\end{tabular}

Fig.1 Biplot graphical analysis of stability and superiority indexes of genotypes 2018-19

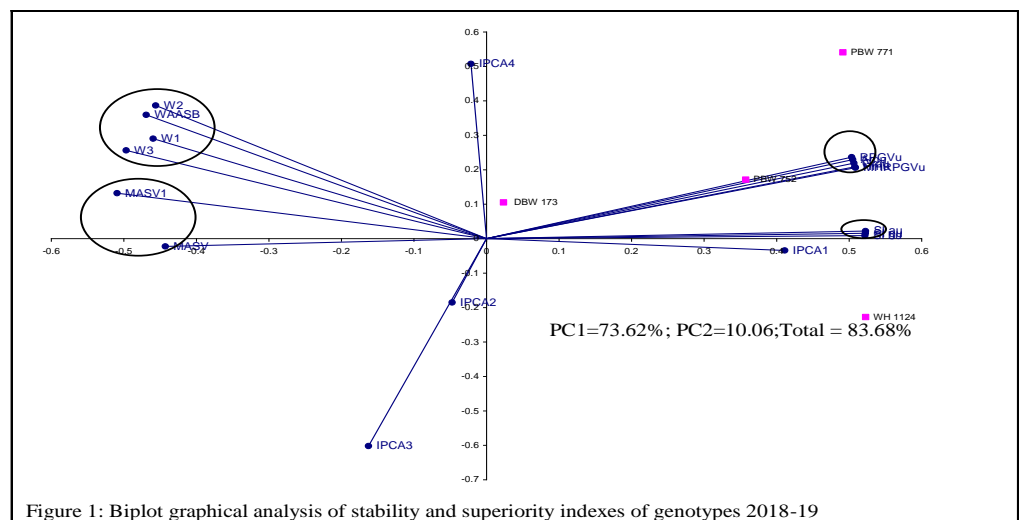


Fig.2 Biplot graphical analysis of stability and superiority indexes of genotypes 2019-20

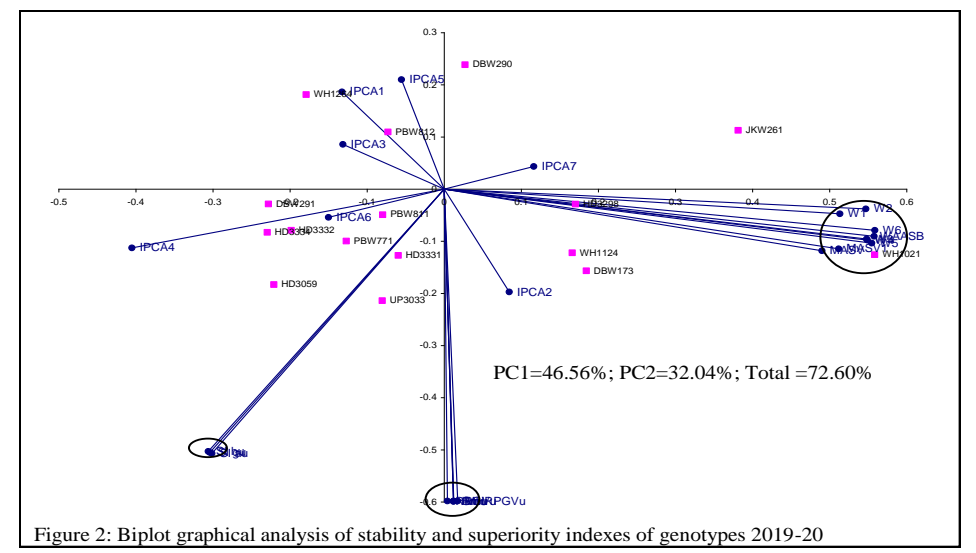

While assigning 65 and 35 weights to average yield (AM) and stability, the Superiority index pointed out WH1124, PBW771 genotypes would maintain high yield and stable performance. SI measure considered GM and stability, selected PBW771, PBW52 genotypes. Values of SI, using $\mathrm{HM}$ and stability, favoured the same set of wheat genotypes PBW771, PBW752. Analytic measures of adaptability RPGV and MHRPGV pointed out PBW771, PBW52 would be more adaptable genotypes.

\section{Biplot analysis of measures}

The first two significant PC's has explained $83.6 \%$ of the total variation (Table 7) with respective contributions of $73.6 \& 10.1$ by PC1\& PC2 (Mohammadi et al., 2015). Two nearby clusters comprised of MASV, MASV1 \& other stability measures with a different number of interaction principal components (Figure 1). Adaptability measures as per arithmetic, geometric and harmonic means along with the corresponding values of RPGV $\&$ MHRPGV expressed bondage with each other and placed in a different quadrant of biplot graphical analysis. However, this group maintained an obtuse angle with stability measures. The cluster of Superiority indexes as per various averages of genotypes yield seen in the same quadrant. The performance difference of genotypes would be very less as per Superiority indexes and adaptability measures.

\section{Second-year of study (2019-20)}

AMMI analysis observed highly significant effects of the environment (E), GxE interaction, and genotypes $(G)$. The diversity of environmental effects was judged by $39.2 \%$ of the total sum of squares due to treatments explained by environments only (Table 3). Genotypes explained $11.8 \%$ of the total sum of squares, whereas GxE interaction explained $35.6 \%$ of treatment variation in yield. First seven multiplicative terms of GxE interaction explained $88.7 \%$ of the sum of squares and little left was residual.

\section{Ranking of genotypes vis-à-vis number of IPCA's}

The ranking of genotype as per absolute IPCA-1 scores were HD3331, HD3332, HD3334 (Table 8). While for IPCA-2, genotypes PBW811, WH1124, PBW771 would be of choice. Values of IPCA-3 favoured UP3033, PBW813, WH1264 wheat genotypes. As per IPCA-4, PBW813 PBW771, DBW291genotypes would be of 
stable performance. WH1264, PBW811, DBW290 genotypes pointed by IPCA-5 measure. Genotypes PBW771, HD3059, HD3298 identified by absolute values of IPCA-6. Lastly, IPCA-7 settled for DBW291, WH1124, DBW290 genotypes for the studied locations of the zone. Analytic measures of adaptability MASV and MASV1consider all significant IPCAs of the analysis. Values of MASV1 identified genotypes DBW291, HD3334, and PBW812 would express stable yield whereas genotypes DBW291, PBW812 \&HD3334 be of stable performance by MASV measure respectively.

Genotype preferences vary as HD3331, HD3332, HD3334, HD3059 based on W1 whereas HD3334, HD3059, HD3332, WH1264 as per W2 values while HD3059, HD3334, WH1264, HD3332 by values of W3 (Table 9). Genotypes HD3059, DBW291, HD3334, PBW813were pointed by W4; W5 favoured DBW291, WH1264, HD3059, HD3334, and lastly by W6 measure DBW291, HD3059, WH1264, HD3059 genotypes would be of choice. Stability measure WAASB based on all significant IPCA's settled for DBW291 WH1264 HD3334 HD3059 genotypes for considered locations of the zone for stable high yield. It is observed that the genotype ranking was altered by the extent to which IPCAs are included in the WAASB estimation. This reinforces the benefits of using the WAASB index since it captures the variations of all IPCAs to compute the stability.

\section{Stable Productive genotypes by AMMI \& BLUP tools}

Mean yield selected UP3033, HD3059, DBW173, WH1021 wheat genotypes (Table 10). Geometric mean observed UP3033, HD3059, DBW173, WH1021, were topranked genotypes. Harmonic Mean of yield expressed higher values for UP3033,
DBW173, HD3059, WH1021 genotypes. Values of HMRPGV ranked UP3033 DBW173, HD3059, WH1021 the performance of the genotypes among the locations. Relative Performance of Genotypic Values had settled for UP3033, HD3059, DBW173, PBW771wheat genotypes.

While assigning 65 and 35 weights to average yield (AM) and stability, the Superiority index pointed out HD3059, UP3033, HD3334, and PBW771 genotypes would maintain high yield and stable performance. SI measure considered GM and stability, selected HD3059, UP3033, HD3334, and PBW771 genotypes. Values of SI, using HM and stability, favoured the same set of wheat genotypes HD3059, UP3033, HD3334, and PBW771. Analytic measures of adaptability RPGV pointed out UP3033, DBW173, PBW771, and MHRPGV settled for UP3033, DBW173, HD3059, and WH1021 as more adaptable genotypes.

\section{Biplot analysis of measures}

The relationships among different measures of wheat genotypes were expressed in a biplot of PC1 and PC2 (Table11). The first two PCs accounted for $72.6 \%(\mathrm{PC} 1=46.5 \%$ and $\mathrm{PC} 2$ $=32 \%)$ of the total variation in the original variables. Stability measures as per utilization of the number of Interaction Principal Components were clustered with AMMI based MASV \& MASV1 measures (Figure 2). Arithmetic, geometric, harmonic means along with corresponding analytic measures of adaptability were clustered together in one group. Moreover, the measures of this group expressed nearly right angle to MASV, MASV1\& stability indexes. Superiority indexes were placed together in one group in a different quadrant. The performance of genotypes as per SI measures will not corroborate with genotypes behaviour based on stability, MASV \& MASV1 measures. 
Superiority Index measures considering 65/35 ratio to yield of genotypes by arithmetic, geometric, harmonic means, and stability by WAASB measures had maintained the distances from measures based on AMMI or WAASB criterion.

In conclusion the GxE interaction study in multi-environment trials had been carried out by a well-established AMMI model. The simultaneous consideration of stability measures and yield would be more appropriate to recommend high-yielding stable wheat genotypes. In the present study, the main advantages of AMMI and BLUP had been combined to increase the reliability of multi-locations trials analysis. An additional advantage was provided by Superiority Indexes to assign variable weights to the yield and stability performance. Depending upon the goal of crop breeding trials, the researchers may prioritize the productivity of a genotype rather than its stability (and viceversa). The stability index of genotype performance has the potential to provide reliable estimates of stability in future studies along with a joint interpretation of performance and stability in biplots while considering the number of significant IPCA's.

\section{Acknowledgments}

The wheat genotypes were evaluated at research fields at coordinated centers of AICW\&BIP across the country. The first author sincerely acknowledges the hard work of all the staff for field evaluation and data recording of wheat genotypes.

\section{Conflict of interest}

The authors declared no conflict of interests.

\section{References}

Agahi K, Ahmadi J, Oghan H A, Fotokian M H, Orang S F 2020. Analysis of genotype $\times$ environment interaction for seed yield in spring oilseed rape using the AMMI model. Crop Breeding and Applied Biotechnology 20(1): e26502012

Ajay B C, Aravind J, Fiyaz R A, Kumar N, Lal C, Kona P, Dagla M C, Bera S K 2019. Rectification of modified AMMI stability value (MASV). Indian J Genet 79(4): 726-731

Ajay BC, Bera SK, Singh AL, Kumar N, Gangadhar K, Kona P 2020. Evaluation of Genotype $\times$ Environment Interaction and Yield Stability Analysis in Peanut Under Phosphorus Stress Condition Using Stability Parameters of AMMI Model. Agric Res 9: 477-486

Annicchiarico P, 1992. Cultivar adaptation and recommendation from alfalfa trials in northern Italy. Journal of Genetics and Plant Breeding 46: 269-278

Ashwini KVR, Ramesh S, Sunitha NC,2021. Comparative BLUP, YREM-based performance and AMMI model-based stability of horse gram [Macrotylom auniflorum (Lam.) Verdc.] genotypes differing in growth habit. Genet Resour Crop

Evolhttps://doi.org/10.1007/s10722020-01089-x

Bocianowski J, Niemann J, NowosadK, 2019. Genotype-by environment interaction for seed quality traits in interspecific cross-derived Brassica lines using additive main effects and multiplicative interaction model. Euphytica 215(7):113

FarshadfarE, 2008. Incorporation of AMMI stability value and grain yield in a single non-parametric index (GSI) in bread wheat. Pak J BiolSci 11:17911796

Farshadfar E, Mahmodi N and Yaghotipoor A, 2011. AMMI stability value and simultaneous estimation of yield and yield stability in bread wheat (Triticum 
aestivum L.). Aust J Crop Sci 5:18371844

GauchHG, 2013. A simple protocol for AMMI analysis of yield trials. Crop Sci 53:1860-1869

Kang MS, 1993. Simultaneous selection for yield and stability in crop performance trials: Consequences for growers. Agronomy Journal 85:754-757

Lin CS and BinnsMR, 1988. A superiority measure of cultivar performance for cultivar $\mathrm{x}$ location data. Canadian Journal of Plant Science 68: 193-198

Mendes F F, Guimarães L J M, Souza J C, Guimarães P E O, Pacheco C A P, Machado J R de A, Meirelles W F, Silva A R da, Parentoni S N, 2012. Adaptability and stability of maize varieties using mixed model methodology. Crop Breeding and Applied Biotechnology 12(2): 111-117

Mohammadi M, Sharifi P, Karimizadeh R, Jafarby JA, Khanzadeh H, Hosseinpour T, Poursiabidi MM, Roustaii M, Hassanpour HM, Mohammadi P, 2015. Stability of grain yield of durum wheat genotypes by AMMI model. Agric For 61(3): 181-193

Mohammadi R, Amri A, 2008. Comparison of parametric and non-parametric methods for selecting stable and adapted durum wheat genotypes in variable environments. Euphytica 159: 419-432

Olivoto T, Lucio A Dal'Col, Gonzalez, Silva JA da, Marchioro VS, 2019. Mean performance and stability in multienvironment trials I: Combining features of AMMI and BLUP techniques. Agron J 111:1-12

Oyekunle M, Menkir A, Mani H, Olaoye G, Usman IS, Ado S, 2017. Stability analysis of maize cultivars adapted to tropical environments using AMMI analysis. Cereal Res Commun 45:336345

Piepho HP, Mo"hring J, Melchinger AE, Bu"chseA, 2008. BLUP for phenotypic selection in plant breeding and variety testing. Euphytica 161(1):209-228

Rao AR and Prabhakaran VT, 2005. Use of AMMI in simultaneous selection of genotypes for yield and stability. Journal of the Indian Society of Agricultural Statistics 59:76-82

ResendeMDV, 2007. Software Selegen REML/BLUP: sistemaestatístico e seleçãogenética computadorizada via modeloslinearesmistos. Embrapa Florestas, Colombo, 350p.

Resende MDV, Duarte JB, 2007. Precision and Quality Control in Variety Trials. PesquisaAgropecuaria Tropical 37: 182194

Zali H, Farshadfar E, Sabaghpour SH, Karimizadeh R 2012. Evaluation of genotype $\times$ environment interaction in chickpea using measures of stability from AMMI model. Ann Biol Res 3:3126-3136

\section{How to cite this article:}

Ajay Verma and Singh, G. P. 2021. Stability, Adaptability Analysis of Wheat Genotypes by AMMI with BLUP for Irrigated Late Sown Trials in North Western Plains Zone. Int.J.Curr.Microbiol.App.Sci. 10(02): 875-887. doi: https://doi.org/10.20546/ijcmas.2021.1002.103 